\title{
The evidence debate broadens: three examples
}

Dr. David McQueen is a senior biomedical research scientist and associate director for Global Health Promotion at the National Center for Chronic Disease Prevention and Health Promotion (NCCDPHP), at the Centers for Disease Control and Prevention (CDC) in Atlanta, Georgia

In recent years the notion of evidence has entered fully into the discussion of practice in public health. It is now widely accepted that evaluation in health promotion and public health should be able to provide evidence that a program, intervention, or strategy is or has been effective in producing changes in a desired direction. Nonetheless, agreed upon definitions of how evidence should be recognized and indeed what should constitute evidence remain somewhat elusive. The field of discussion has become fuzzier as the debate on evidence has both deepened and broadened. Earlier confidence in the randomized clinical trials (RCT) as the sine qua non of the proof of effectiveness has given way to numerous efforts to define the nature of what is needed to show that a program is effective and what is a theoretical basis for this. Furthermore, the areas of public health effort considered amenable to the evidence debate have broadened, so that now we want proof of the effectiveness of policies, of strategies, and multi-level intervention programs. Finally, the methods needed to demonstrate effectiveness and provide evidence have broadened considerably. The three papers in the special section of this issue illustrate these three aspects.

The paper by Rütten et al illustrates how theory is critical in assessing the determinants of health policy impact. The issue taken up is how health policy ultimately relates to evidence. What seems to be understood in everyday discourse on policy is that evidence plays a very small role in decision taking by policy makers. The authors note this and begin to offer the foundations for a framework that takes into account evidence for effective health promotion policy development. Their quest is to identify the key determinants of health policy that theoretically lead to an understanding of policy effectiveness. They allow for the importance of a logical framework and this represents one of the widely recognized key components of current thinking about illustrating evidence, namely the construction of some type of logic model. The authors construct their own unique model based on the logic of events. This is the type of approach that needs to be refined and elaborated as the evidence discussion develops.

On a less theoretical and more pragmatic basis, Thurston et al. search for a testable framework for assessing the effectiveness of health promotion. This framework undertook the task of developing a framework sensitive to both qualitative and quantitative approaches, more in keeping with what the actual field of health promotion practice looked like. This is an example of effectiveness assessment operating in the real world of small but numerous health promotion projects. The authors developed a framework to assess some 180 health promotion projects in Canada. In an assessment of effectiveness one almost always faces the task of what criteria are to be used to judge projects as they actually have occurred and based on whatever records, in whatever form, exist. Thus they present one example of such an undertaking.

Finally, Michel O'Neill provides a critical paper that asks some very fundamental questions about evidence, the search for it, and why it is a search that is necessary for the field of health promotion. This discussion introduces fundamental questions about the nature of knowledge and how a field such as health promotion can learn about the world without being restricted to the narrow criteria that have been applied in many of the biomedical sciences.

In brief, these three articles provide an excellent excursion into some of the key issues that now occupy those involved in what I have termed the "evidence debate."

Soz.- Präventivmed. 48 (2003) 275-276 
(McQueen, 2002). Key issues remain around the definition and distinction of such terms as "impact", "effectiveness,"

"health promotion," "best practices" and a number of key, but rather loosely defined, terms that are widely used.

Despite the volume of literature generated, there remain key epistemological questions to be addressed.

\section{David V. McQueen}

Reference

McQueen DV (2002). The evidence debate.

J Epidemiol Community Health 56: 83-4.
Address for correspondence

\author{
Prof. Dr. David V. McQueen \\ CDC/NCCDPHP \\ Office of the Director MS K40 \\ 4770 Buford Highway NE \\ USA-Atlanta 30341 \\ e-mail: dvmcqueen@cdc.gov
}

